\title{
Influencia de la temperatura en el diseño de mezclas asfálticas tibias con aceite de palma industrial y artesanal de Yurimaguas
}

\section{Influence of temperature on the design of warm mixtures asphalt with industrial and artisanal palm oil from Yurimaguas}

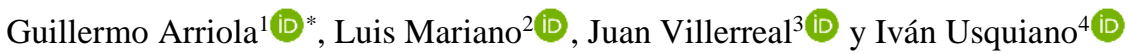

\begin{abstract}
RESUMEN
La producción actual de mezclas asfálticas en caliente para carreteras, aportan grandes cantidades de gases tóxicos nocivos para el medio ambiente debido a las altas temperaturas durante su preparación, por lo que se necesitan tomar medidas correctivas que sean sostenibles y económicas. Éstas medidas pueden verse reflejadas durante la elaboración de mezclas con la inclusión de otros materiales como aceites, polímeros o plásticos que influyan en la disminución de la temperatura. En la investigación se optó por incorporar un aceite crudo de palma de una planta de origen africano muy usado en la ciudad de Yurimaguas, Perú. Los aceites que se emplearon fueron del tipo industrial y artesanal a una temperatura de mezcla máxima de $130^{\circ} \mathrm{C}$. Las variaciones de temperatura aceptables estuvieron en el rango de $128^{\circ} \mathrm{C}$, $125,50^{\circ} \mathrm{C}, 120^{\circ} \mathrm{C}, 117,50^{\circ} \mathrm{C}$ y $115^{\circ} \mathrm{C}$, considerando en todos los casos los requerimientos de laboratorio. Los resultados obtenidos indican que el aceite crudo de palma si influye en el diseño de mezclas asfálticas de hasta $115^{\circ} \mathrm{C}$ bajando considerablemente en $45^{\circ} \mathrm{C}$ respecto a una mezcla asfáltica en caliente típica, sin perder de lado la óptima relación estabilidad y flujo.
\end{abstract}

Palabras clave: Carreteras, estabilidad, flujo, materiales

\begin{abstract}
The current production of hot asphalt mixes for road works worldwide contributes large amounts of toxic gases that are harmful to the environment due to the high temperatures during their preparation, so corrective measures need to be taken that are sustainable and economical. These measures can be reflected during the elaboration of mixtures with the inclusion of other materials such as oils, polymers or plastics that influence the decrease in temperature. So for the present investigation was decided to incorporate a crude palm oil from a plant of African origin widely used in the city of Yurimaguas in Peru. The oils used were of the industrial and artisanal type at a maximum mixing temperature of $130.00^{\circ} \mathrm{C}$. Acceptable temperature variations were in the range of $128^{\circ} \mathrm{C}, 125,50^{\circ} \mathrm{C}, 120^{\circ} \mathrm{C}, 117,50^{\circ} \mathrm{C}$ and $115^{\circ} \mathrm{C}$, considering in all cases the laboratory requirements. Finally, the results obtained indicate that crude palm oil does influence the design of the mix asphalt up to $115^{\circ} \mathrm{C}$, decreasing considerably by $45^{\circ} \mathrm{C}$ compared to a typical hot mix asphalt, without losing the optimal stability ratio and flow.
\end{abstract}

Keywords: Highway, stability, flow, materials.

DOI: https://doi.org/10.37787/pakamuros-unj.v9i1.158

Recibido: 09/01/2021 Aceptado: 01/02/2021

* Autor para correspondencia

Universidad Señor de Sipán, Perú. Email: arriola2589@gmail.com

Universidad Señor de Sipán, Perú. Email: villegas_luis9@outlook.com

Universidad Señor de Sipán, Perú. Email: broth2408@gmail.com

4. Universidad Señor de Sipán, Perú. Email: usquiano24@gmail.com 


\section{INTRODUCCIÓN}

Las mezclas asfálticas se definen como la combinación de agregados seleccionados uniformemente mezclados y distribuidos, recubiertos a la vez por material bituminoso que permiten una adherencia tal que las proporciones relativas de estos determinan las propiedades físicas y además el rendimiento de la misma como mezcla terminada y que su deterioro no sea a corto plazo, Mardones et al. (2018), explican que las carreteras están expuestas a agentes externos, como la lluvia, radiación solar, el aumento del flujo vehicular, que contribuyen a generar daños en este tipo de pavimentos, además Luo et al. (2019), afirman que también las mezclas asfálticas tienen aplicaciones en la pavimentación de puentes de acero de grandes luces previa evaluación preliminar y una comparación de las condiciones y tecnologías de construcción, sin embargo en la actualidad la gran producción de estas mezclas asfálticas en caliente producen grandes cantidades de gases tóxicos nocivos por lo que se necesitan considerar ciertas medidas y que para Cheraghian et al. (2020) y Mosa et al. (2020), estas consideraciones deben ser seguras, correctivas, sostenibles y económicas, siguiendo una conciencia ecológica. Otra opción importante según Arroyo et al. (2018), las carreteras más sostenibles se pueden evaluar diferentes alternativas utilizando un método de toma de decisiones multicriterio basado en las mejores ventajas de cada una de ellas para su posible elección, en ese sentido diversas investigaciones apuntan hacia el desarrollo de tecnologías limpias, según Garcia et al. (2017) y Alimohammadi et al. (2020), manifiestan que se debe implementar nuevas tecnologías para pavimentos y se debe centrarse en la caracterización y adición bien definida de otros materiales en proporción a la mezcla asfáltica, por ejemplo Daniel (2020), evalúa las bondades de la adhesión de polímeros como un aditivo para producir mezclas asfálticas, tanto por el proceso húmedo como por el proceso seco, concluyendo en que el efecto de esta incorporación en mezclas bituminosas puede combatir el mecanismo de falla en las estructuras del pavimento, sin embargo es necesario ampliar las investigaciones para estudiar y validar como las nuevas tecnologías influyen en el diseño del pavimento para una mejora continua.

Para Castro et al. (2020), se deben elaborar mezclas asfálticas con la adición de diversos materiales amigables que al mezclarse en su totalidad minimicen el impacto ambiental, tal es el caso indicado por Woszuk y Franus (2017) y Woszuk (2018), mencionan sobre el uso de un aditivo como la zeoilta que no implica una inversión costosa para cambiar el método de producción de la mezcla asfáltica cuyo fin es disminuir la temperatura. Otro caso destacable es el de Ziari et al. (2019), que estudiaron el efecto de la escoria de cobre en el desempeño de las mezclas tibias. Hoy en día las mezclas asfálticas tibias comúnmente conocidas en sus siglas en inglés como Warm Mix Asphalt (WMA), tal y como lo sostienen 
Bower et al. (2016), son una tecnología relativamente nueva y emergente para la industria del asfalto y que se debería evaluar su desempeño en comparación con las mezclas asfálticas convencionales. Martin et al. (2019), el asfalto de mezcla tibia se produce a menor temperatura y por lo tanto su producción en la planta de asfalto es favorable en términos de consumo de energía y emisión de $\mathrm{CO}_{2}$. Abed y Thom (2019), las mezclas tibias han sido desarrolladas para minimizar el impacto ambiental del asfalto a partir de la reducción de las temperaturas de producción. Ameri et al. (2020), manifiestan que los estudios demuestran que la tecnología de mezclas asfálticas tibias son una solución práctica contra la alta temperatura de producción de las mezclas asfálticas convencionales. Un uso práctico también de las mezclas de baja temperatura según Chen et al. (2021), indican que las temperaturas de mezcla y compactación de la mezcla asfáltica del tipo termocrómica se pueden reducir gracias a esta tecnología. Por otro lado, Chung et al. (2013), destacan que las mezclas asfálticas tibias son una buena técnica para reducir la temperatura, pero aún es un desafío determinar las temperaturas y compactación de la mezcla de asfalto óptima.

Otro aspecto importante está relacionado a los elementos que se unen a la propia mezcla y como influyen significativamente en la disminución de la temperatura sin que pierda sus propiedades físicas, mecánicas y que según Martinho et al. (2020) puedan resistir a la deformación permanente, fatiga, ahuellamiento, módulo de rigidez, susceptibilidad térmica, desgaste prematuro y envejecimiento, esta última condición es importante. El envejecimiento ocurre durante la fase de mezcla, sin embargo, la parte principal del envejecimiento a corto plazo ocurre durante el almacenamiento de la mezcla de planta y el cambio súbito de la temperatura puede afectar a las propiedades reológicas del asfalto, en ese sentido sostienen además que esta caracterización de la mezcla asfáltica se puede evaluar con el modelo cinético de envejecimiento (Li et al.2019; Song et al.2018; Arafat y Wasiuddin 2019; Liu et al. 2019). También Ayyar et al. (2019), menciona que existen muy pocos datos sobre la influencia de la baja temperatura de las mezclas asfálticas para caracterizar estas condiciones en laboratorio. Por tanto, es importante evaluar y analizar la literatura disponible para comprender mejor las mezclas tibias ya que al agregarle otros agentes externos como los elementos reciclados su rendimiento se podría ver afectado (Hettiarachchi et al. 2019),

Por lo expuesto se busca con la presente investigación evaluar la influencia de la temperatura en el diseño de mezclas asfálticas tibias con aceite de palma industrial y artesanal de la ciudad de Yurimaguas, tratando de determinar el contenido óptimo de asfalto a menor temperatura. 


\section{MATERIALES Y MÉTODOS}

La metodología empleada, se sustenta en lo desarrollado por Lopera y Córdoba (2013). El estudio fue de tipo experimental, y el desempeño del asfalto se evaluó utilizando como técnica los ensayos de laboratorio. Tomando como referencia los procedimientos realizados por Lopera y Córdoba (2013), Rondón et al. (2016) y Usquiano y Villarreal (2018), para la obtención de muestras, se procedió a la extracción de los materiales de cantera disponibles de una planta de procesos ubicada en la vía Dv. Ciudad de Dios - Chilete, lado derecho, luego los especímenes fueron llevados al laboratorio de ensayos y materiales de la Universidad Señor de Sipán para los respectivos controles de calidad, caracterización de los agregados y elaboración de las mezclas asfálticas; se determinaron tres tipos de mezclas asfálticas donde la primera fue una mezcla asfáltica en caliente convencional y las otras dos fueron con la adición de los aceites industrial y artesanal de la ciudad de Yurimaguas.

Para la determinación del mejor porcentaje de asfalto, se realizaron las combinaciones de agregados chancados que previamente se ajustan a las granulometrías propuestas por la normatividad del Ministerio de Transportes y Comunicaciones del Perú obteniéndose un ajuste a la curva granulométrica MAC-2 y las briquetas que fueron realizadas bajo una misma energía de compactación según Mardones et al. (2018) y el método de ensayo Marshall (MTC, 2016).

Finalmente, siguiendo el criterio que sugieren Usquiano y Villarreal (2018), que recomienda emplear relaciones cada 0,50\%, se optó entonces para este caso de estudio un asfalto PEN 60-70 con relaciones de porcentaje de 4,50\%,5\%,5,50\%,6\% y 6,50\% y para la adición del aceite crudo de palma (el industrial y el artesanal) con proporciones de $0,50 \%, 1 \%, 1,50 \%, 2 \%, 2,50 \%$ y $3 \%$, para añadir a la mezcla asfáltica a una temperatura de mezcla máxima de $130^{\circ} \mathrm{C}$, valor de temperatura aceptable según los resultados alcanzados por Vaitkus et al. (2016).

\section{RESULTADOS}

La Tabla 1, indica los resultados logrados que demuestran que la de mejor comportamiento en comparación con la mezcla convencional es la mezcla asfáltica tibia adicionada con aceite crudo de palma industrial cuyo principal parámetro comparativo es el contenido de asfalto. Cabe indicar que para el análisis de la elección del porcentaje de aceite en la mezcla estuvo condicionado por el aumento y/o disminución del porcentaje óptimo de asfalto respecto a la mezcla asfáltica tibia. 
Tabla 1. Comparación de parámetros obtenidos del ensayo Marshall según el tipo de mezcla asfáltica ensayada

\begin{tabular}{|c|c|c|c|c|c|}
\hline \multirow[b]{2}{*}{ Parámetro } & \multirow[b]{2}{*}{ Unidad } & \multirow{2}{*}{$\begin{array}{c}\text { Mezcla asfáltica } \\
\text { en caliente } \\
\text { (convencional) a } \\
160^{\circ} \mathrm{C}\end{array}$} & \multicolumn{2}{|c|}{$\begin{array}{c}\text { Mezcla asfáltica } \\
\text { tibia (WMA) a } 130^{\circ} \mathrm{C}\end{array}$} & \multirow{2}{*}{$\begin{array}{c}\text { Especificación } \\
\text { técnica del } \\
\text { Ministerio de } \\
\text { Transportes y } \\
\text { Comunicaciones } \\
\text { (MTC) } \\
\end{array}$} \\
\hline & & & $\begin{array}{c}\text { Con aceite } \\
\text { de palma } \\
\text { industrial al } \\
\mathbf{3 \%} \\
\end{array}$ & 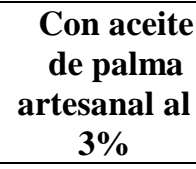 & \\
\hline Óptimo contenido de asfalto & $\%$ & 5,750 & 5,700 & 5,800 & ---- \\
\hline Peso específico & $\mathrm{g} / \mathrm{cm} 3$ & 2,387 & 2,385 & 2,383 & ---- \\
\hline Vacíos de aire & $\%$ & 3,800 & 3,950 & 3,850 & De $3 \%$ a $5 \%$ \\
\hline Vacíos de Agregado Mineral & $\%$ & 15,600 & 15,850 & 16,050 & Mínimo 14\% \\
\hline Vacíos llenos de asfalto & $\%$ & 75,900 & 76,150 & 76,100 & De $65 \%$ a $75 \%$ \\
\hline Flujo & $\mathrm{mm}$ & $3 ., 00$ & 3,250 & 3,150 & De $2 \mathrm{~mm}$ a $4 \mathrm{~mm}$ \\
\hline Estabilidad & Lbs & 1355,400 & 1359,500 & 1375,200 & Mínimo 815 Lbs \\
\hline
\end{tabular}

La Figura 1 muestra las curvas del comportamiento mecánico de la mezcla asfáltica tibia elegida para el diseño con la adición del aceite de palma industrial al 3\%, tanto para la estabilidad y el flujo.

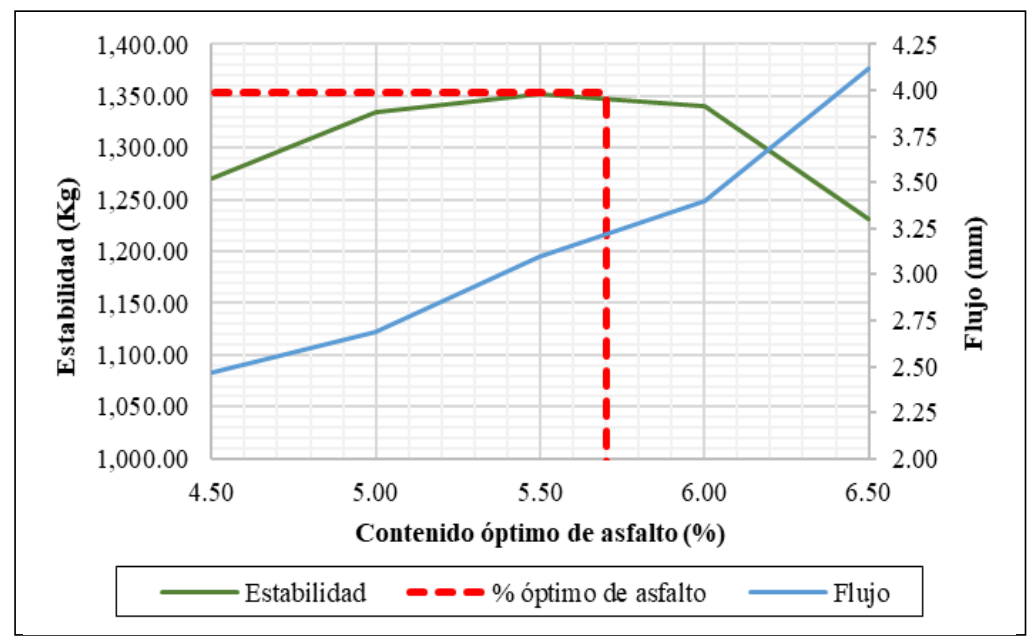

Figura 1. Curvas de estabilidad - flujo - \% óptimo de asfalto para el aceite industrial.

Luego de haber elegido la mezcla asfáltica tibia con aceite industrial más desfavorable al 3\%, se procedió a evaluar las variaciones de temperatura menores a la mezcla patrón, las temperaturas aceptables estuvieron en el rango de $128^{\circ} \mathrm{C}, 125,50^{\circ} \mathrm{C}, 120^{\circ} \mathrm{C}, 117,50^{\circ} \mathrm{C}$ y $115^{\circ} \mathrm{C}$ y a partir de lo señalado por Yang et al. (2020) se consideró un control de contenido de humedad ya que los agregados no se pueden secar completamente debido a la baja temperatura de calentamiento, lo que afecta directamente el desempeño del pavimento. La Tabla 2, indica los resultados de las temperaturas admisibles para la mezcla asfáltica tibia con aceite de palma industrial, donde se destacan los contenidos óptimos de asfalto iguales y menores del 6\%, con una incorporación del 3\% de aceite. 
Tabla 2. Valores de incorporación de aceite, contenido de asfalto y promedio de contenido de asfalto

\begin{tabular}{|c|c|c|c|c|}
\hline $\begin{array}{l}\text { Tipo de } \\
\text { mezcla }\end{array}$ & $\begin{array}{c}\text { Temperatura } \\
\left({ }^{\circ} \mathbf{C}\right)\end{array}$ & $\begin{array}{l}\text { Incorporación } \\
\text { de aceite }(\%)\end{array}$ & $\begin{array}{l}\text { Óptimo contenido } \\
\text { de asfalto }(\%)\end{array}$ & $\begin{array}{l}\text { Promedio del óptimo } \\
\text { contenido de asfalto }(\%)\end{array}$ \\
\hline \multirow{30}{*}{$\begin{array}{c}\text { Mezcla } \\
\text { asfáltica } \\
\text { tibia con } \\
\text { adición de } \\
\text { aceite } \\
\text { industrial }\end{array}$} & \multirow{6}{*}{115,00} & 0,50 & 6,75 & \multirow{6}{*}{6,34} \\
\hline & & 1,00 & 6,55 & \\
\hline & & 1,50 & 6,40 & \\
\hline & & 2,00 & 6,20 & \\
\hline & & 2,50 & 6,15 & \\
\hline & & 3,00 & 6,00 & \\
\hline & \multirow{6}{*}{117,50} & 0,50 & 6,55 & \multirow{6}{*}{6,28} \\
\hline & & 1,00 & 6,50 & \\
\hline & & 1,50 & 6,45 & \\
\hline & & 2,00 & 6,30 & \\
\hline & & 2,50 & 6,00 & \\
\hline & & 3,00 & 5,85 & \\
\hline & \multirow{6}{*}{120,00} & 0,50 & 6,50 & \multirow{6}{*}{6,26} \\
\hline & & 1,00 & 6,45 & \\
\hline & & 1,50 & 6,30 & \\
\hline & & 2,00 & 6,20 & \\
\hline & & 2,50 & 6,10 & \\
\hline & & 3,00 & 6,00 & \\
\hline & \multirow{6}{*}{125,50} & 0,50 & 6,20 & \multirow{6}{*}{5,98} \\
\hline & & 1,00 & 6,00 & \\
\hline & & 1,50 & 6,00 & \\
\hline & & 2,00 & 5,90 & \\
\hline & & 2,50 & 5,90 & \\
\hline & & 3,00 & 5,85 & \\
\hline & \multirow{6}{*}{128,00} & 0,50 & 6,00 & \multirow{6}{*}{5,80} \\
\hline & & 1,00 & 5,85 & \\
\hline & & 1,50 & 5,85 & \\
\hline & & 2,00 & 5,80 & \\
\hline & & 2,50 & 5,70 & \\
\hline & & 3,00 & 5,60 & \\
\hline
\end{tabular}

\section{DISCUSIÓN}

En concordancia con los resultados logrados por Lopera y Córdoba (2013), Rondón et al. (2016) y Usquiano y Villarreal (2018), se realizaron los ensayos de laboratorio y controles de calidad para los agregados que se utilizaron en la elaboración de las mezclas asfálticas permitiendo verificar los requisitos mínimos y tolerancias máximas exigidas por la normativa de carreteras del Perú (MTC, 2016). Sin embargo, no existe en nuestro país algún manual, normatividad y/o reglamento sobre la incorporación de aceites crudo de palma en el diseño de mezclas asfálticas, tampoco sobre los controles de temperatura 
con la adición de aceites de palma, además para Garcia et al. (2017), los rendimientos generales de las mezclas tibias se pueden mejorar si la selección de materiales se optimiza y esto a su vez permita un adecuado enlace entre los agregados y el asfalto.

De los resultados obtenidos, la mezcla asfáltica tibia elaborada logró una reducción de hasta $45^{\circ} \mathrm{C}$ de temperatura en comparación con la mezcla asfáltica en caliente, sin embargo no se mantuvo constante el contenido óptimo de asfalto para todas las incorporaciones del aceite a la mezcla tibia tal y como se detalla en la Tabla 2, este efecto se puede deber a lo señalado por Chung et al. (2013), ya que las mezclas tibias reducen la temperatura de $20^{\circ} \mathrm{C}$ a $30^{\circ} \mathrm{C}$, para Rahman et al. (2020), mencionan que la mezcla tibia se mezcla y compacta comúnmente de $15^{\circ} \mathrm{C}$ a $30^{\circ} \mathrm{C}$ por debajo del asfalto de una mezcla asfáltica en caliente convencional y en el caso de Ameri et al. (2020), redujeron las temperaturas de mezcla y compactación en un rango de $20^{\circ} \mathrm{C}$ a $25^{\circ} \mathrm{C}$.

Luego por lo expuesto y según los ensayos realizados se recomienda una temperatura ideal de $128^{\circ} \mathrm{C}$ con una adición de $3 \%$ con lo cual se obtendrá un 5,60\% de contenido de asfalto óptimo, pero no se descarta en su totalidad una temperatura mínima de $115^{\circ} \mathrm{C}$ con un porcentaje de aceite de $3 \%$ a pesar de tener contenido de asfalto de 6\%, el cual está por encima del óptimo de la mezcla convencional, ya que Abed y Thom (2019) en su investigación obtuvieron como resultados que los aditivos en la mezcla tibia pueden mejorar el recubrimiento de agregados incluso cuando se mezclan a $115^{\circ} \mathrm{C}$.

\section{CONCLUSIONES}

Se aplicó el procedimiento de ensayo Marshall para caracterizar el comportamiento mecánico de las mezclas asfálticas empleando para ello briquetas que determinaron el peso específico, vacíos de aire, vacíos de agregado mineral, vacíos llenos de asfalto, flujo, estabilidad, óptimo contenido de asfalto y porcentajes de adición del aceite de palma industrial y artesanal, con lo cual se estableció que el contenido de aceite patrón es de 3\% para el mejor desempeño de las mezclas tibias la cual fue la del tipo industrial. Se determinaron cinco rangos de temperaturas para la mezcla tibia elegida, concluyendo en que la más adecuada es la de temperatura de $128^{\circ} \mathrm{C}$ para un porcentaje de adición de aceite de palma industrial de $3 \%$ con el que se obtuvo un óptimo contenido de asfalto de 5,60\%, pero no se descartan los demás rangos de temperatura conseguidos con la presente investigación puesto que no se pierden las óptimas relaciones de estabilidad y flujo hasta un mínimo de $115^{\circ} \mathrm{C}$.

\section{REFERENCIAS BIBLIOGRÁFICAS}


Abed, A., \& Thom, N. G. (Marzo de 2019). A novel approach for rational determination of warm mix asphalt production temperatures. Construction and Building Materials, 200, 80-93. doi:10.1016/j.conbuildmat.2018.12.082

Alimohammadi, H., Zheng, J., Buss, A., Schaefer, V., Williams, C., \& Zheng, G. (Diciembre de 2020). Field and simulated rutting behavior of hot mix and warm mix asphalt overlays. Construction and Building Materials, 265. doi:10.1016/j.conbuildmat.2020.120366

Ameri, M., Yazdipanah, F., Rahimi, A., \& Afshin, A. (Agosto de 2020). Production temperatures and mechanical performance of rubberized asphalt mixtures modified with two warm mix asphalt (WMA) additives. Materials and Structures/Materiaux et Constructions, 53(4). doi:10.1617/s11527-020-01542-4

Arafat, S., \& Wasiuddin, N. (Noviembre de 2019). Understanding the short-term aging of warm mix asphalt using rolling thin film oven. International Journal of Pavement Research and Technology, 12(6), 638-647. doi:10.1007/s42947-019-0076-2

Arroyo, P., Herrera, R., Salazar, L., Giménez, Z., Martínez, J., \& Calahorra, M. (Diciembre de 2018). A new approach for integrating environmental, social and economic factors to evaluate asphalt mixtures with and without waste tires. Revista Ingeniería de Construcción, 33(3), 301-314. doi:10.4067/S0718-50732018000300301

Ayyar, P., Lakshmi, S., Padmarekha, A., \& Krishnan, J. (Noviembre de 2019). Influence of rest period on the fatigue response of bituminous mixture at low temperature. International Journal of Pavement Research and Technology, 12(6), 674-681. doi:10.1007/s42947-019-0080-6

Behnood, A. (Junio de 2020). A review of the warm mix asphalt (WMA) technologies: Effects on thermo-mechanical and rheological properties. Journal of Cleaner Production, 259. doi:10.1016/j.jclepro.2020.120817

Bower, N., Wen, H., Wu, S., Willoughby, K., Weston, J., \& DeVol, J. (2016). Evaluation of the performance of warm mix asphalt in Washington state. International Journal of Pavement Engineering, 17(5), 423-434. doi:10.1080/10298436.2014.993199

Castro, H., Romero, B., Vásquez, C., \& Arriola, G. (Diciembre de 2020). Influencia de la cáscara y fibra de coco en mezclas asfálticas en caliente. Revista Científica Ingeniería: Ciencia, Tecnología e Innovación, 7(2), 176-190. doi:10.26495/icti.v7i2.1506 
Chen, Z., Zhang, H., Duan, H., \& Shi, C. (Enero de 2021). Improvement of thermal and optical responses of short-term aged thermochromic asphalt binder by warm-mix asphalt technology. Journal of Cleaner Production, 279. doi:10.1016/j.jclepro.2020.123675

Cheraghian, G., Cannone, A., You, Z., Chen, S., Kim, Y., Westerhoff, J., . . Wistuba, M. (Setiembre de 2020). Warm mix asphalt technology: An up to date review. Journal of Cleaner Production, 268. doi:10.1016/j.jclepro.2020.122128

Chung, W., Hao, P. R., Zhang, X., \& Adhikari, S. (Noviembre de 2013). Determination of the production temperature of warm mix asphalt by workability test. Construction and Building Materials, 48, 1165-1170. doi:10.1016/j.conbuildmat.2013.07.097

Daniel, G. (Noviembre de 2020). Assessing the impact of the incorporation of aramid and polyolefin to hot and warm asphaltic mixture using dry and wet process: A Review. IOP Conference Series: Materials Science and Engineering, 930(1). doi:10.1088/1757-899X/930/1/012062

Garcia, L., Kassem, E., Little, D., \& Masad, E. (2017). Fundamental evaluation of moisture damage in warm-mix asphalts. Road Materials and Pavement Design, 18(1 / supplement), 258-283. doi:10.1080/14680629.2016.1266765

Hettiarachchi, C., Hou, X., Wang, J., \& Xiao, F. (Diciembre de 2019). A comprehensive review on the utilization of reclaimed asphalt material with warm mix asphalt technology. Construction and Building Materials, 227. doi:10.1016/j.conbuildmat.2019.117096

Lei, J., Zheng, N., Xu, X., Wu, C., \& Lü, D. (Agosto de 2020). High Temperature Rheological Properties of Warm Mixed Asphalt. Jianzhu Cailiao Xuebao/Journal of Building Materials, 23(4), 904-911. doi:10.3969/j.issn.1007-9629.2020.04.024

Li, Q., Zhang, Z., \& Zhang, S. (Julio de 2019). Performance evaluation of warm-mix recycled asphalt binders after long-term aging. Journal of Testing and Evaluation, 47(4), 16. doi:10.1520/JTE20180157

Liu, X., Li, B., Jia, M., Zhang, Z., Liu, Z., \& Li. (Diciembre de 2019). Influence of short-term aging on anti-cracking performance of warm modified asphalt at intermediate temperature. Colloids and Surfaces A: Physicochemical and Engineering Aspects, 582. doi:10.1016/j.colsurfa.2019.123877

Lopera, C., \& Córdoba, J. (Mayo de 2013). Diseño de mezcla asfáltica tibia a partir de la mezcla de asfalto y aceite crudo de palma. DYNA, 80(179), 99-108. Obtenido de https://revistas.unal.edu.co/index.php/dyna/article/view/28196/40654 
Luo, S., Liu, Z., Yang, X., Lu, Q., \& Yin, J. (Diciembre de 2019). Construction technology of warm and hot mix epoxy asphalt paving for long-span steel bridge. Journal of Construction Engineering and Management, 145(12). doi:10.1061/(ASCE)CO.1943-7862.0001716

Mardones, L., Sánchez, E., Calabi, A., \& Valdés, G. (2018). Evaluación de las propiedades mecánicas de mezclas asfálticas con la incorporación de fibras sintéticas de aramida y polipropileno. Revista Infraestructura Vial, 20(36), 15-24. doi:10.15517/IV.V20I36.37729

Martin, H., Kerstin, Z., \& Joachim. (Julio de 2019). Reduced emissions of warm mix asphalt during construction. Road Materials and Pavement Design, 20(2), S568-S577. doi:10.1080/14680629.2019.1628426

Martinho, F., Picado-Santos, L., \& Capitão, S. (Agosto de 2020). Assessment of warm-mix asphalt concrete containing sub-products as part of aggregate blend. International Journal of Pavement Engineering, 21(10), 1213-1222. doi:10.1080/10298436.2018.1533135

Mosa, A., Salem, L., \& Waryosh, W. (Octubre de 2020). New admixture for foamed warm mix asphalt: A comparative study. Iranian Journal of Science and Technology - Transactions of Civil Engineering, 44, 649-660. doi:10.1007/s40996-020-00397-7

MTC. (2016). Norma MTC E-504: Resistencia de mezclas bituminosas empleando el aparato Marshall. Ministerio de Tranportes y Comunicaciones. Lima: Dirección General de Caminos y Ferrocarriles del Ministerio de Tranportes y Comunicaciones. Obtenido de http://transparencia.mtc.gob.pe/idm_docs/normas_legales/1_0_3729.pdf

Rahman, T., Dawson, A., \& Thom, N. (Junio de 2020). Warm mix asphalt (WMA) for rapid construction in airfield pavement. Construction and Building Materials, 246. doi:10.1016/j.conbuildmat.2020.118411

Rondón, H., Ocampo, M., Vacca, H., Reyes, F., Nieto, J., \& Beltrán, D. (Diciembre de 2016). The mechanical behavior of two warm-mix asphalts. Revista Ingeniería e Investigación, 36(3), 29-38. doi:10.15446/ing.investig.v36n3.54861

Song, Y., Ding, N., Liu, H., Lin, M., \& Cheng, H. (Febrero de 2018). Effect of warm mix agents type and dosage on rheological property of different asphalt. Fuhe Cailiao Xuebao/Acta Materiae Compositae Sinica, 35(2), 451-459. doi:10.13801/j.cnki.fhclxb.20170608.001

Usquiano, I., \& Villarreal, J. (Junio de 2018). Diseño de una mezcla asfáltica tibia con aceite crudo de palma. Ignosis, 4(1), 41-47. doi:10.18050/ingnosis.v4i1.2060 
Vaitkus, A., Čygas, D., Laurinavičius, A., Vorobjovas, V., \& Perveneckas, Z. (Junio de 2016). Influence of warm mix asphalt technology on asphalt physical and mechanical properties. Construction and Building Materials, 112, 800-806. doi:10.1016/j.conbuildmat.2016.02.212

Wang, C., Hao, P., \& Ruan, F. (Agosto de 2020). Method for determining mixing and compaction temperature of warm mix asphalt. Wuhan Ligong Daxue Xuebao (Jiaotong Kexue Yu Gongcheng Ban)/Journal of Wuhan University of Technology (Transportation Science and Engineering), 44(4), 640-643. doi:10.3963/j.issn.2095-3844.2020.04.010

Woszuk, A. (Agosto de 2018). Application of fly ash derived zeolites in warm-mix asphalt technology. Materials, 11(9). doi:10.3390/ma11091542

Woszuk, A., \& Franus, W. (2017). A review of the application of zeolite materials in warm mix asphalt technologies. Applied Sciences (Switzerland), 7(3). doi:10.3390/APP7030293

Yang, B., Xu, H., Zhou, P., \& Tan, Y. (Setiembre de 2020). Investigation of aggregate moisture content variation and its impact on pavement performance of WMA. Construction and Building Materials, 225. doi:10.1016/j.conbuildmat.2020.119350

Ziari, H., Moniri, A., Imaninasab, R., \& Nakhei, M. (Julio de 2019). Effect of copper slag on performance of warm mix asphalt. International Journal of Pavement Engineering, 20(7), 775-781. doi:10.1080/10298436.2017.1339884 